\title{
Two-loop effective potential in noncommutative scalar field theory
}

\author{
Wung-Hong Huang \\ Department of Physics \\ National Cheng Kung University \\ Tainan,70101,Taiwan
}

\begin{abstract}
The renormalization of effective potential for the noncommutative scalar field theory is investigated to the two-loop approximation. It is seen that the nonplanar diagram does not appear in the one-loop potential. However, nonplanar diagram can become dominant in the two-loop level as the noncommutativity of geometry is sufficiently small. The result shows that the radiative corrections from the nonplanar diagrams have an inclination to induce the spontaneously symmetry breaking if it is not broken in the tree level, and have an inclination to restore the symmetry breaking if it has been broken in the tree level.
\end{abstract}

E-mail: whhwung@mail.ncku.edu.tw

Typeset by Latex 


\section{Introduction}

Noncommutative field theories have been received a great deal of attention [1-4]. Historically, it is a hope that the deformed geometry in the small spacetime would be possible to cure the quantum-field divergences, especially in the gravity theory. The work of Filk [5], however, proved that the noncommutative theory exhibits the same divergence as the commutative one.

The renovation of the interesting in noncommutative field theories is that it have proved to arise naturally in the string/M theories [6-9]. Initially, Connes, Douglas and Schwarz [6] had shown that the supersymmetric gauge theory on noncommutative torus is naturally related to the compactification of Matrix theory [10]. More recently, it is known that the dynamics of a D-brane in the presence of a B-field can, in certain limits, be described by the noncommutative field theories [9].

The quantum aspects of the noncommutative field theories have been pursued via perturbative analysis over diverse model [11-16]. A distinct characteristic of the noncommutative field theories, found by Minwalla, Raamsdonk and Seiberg [13], is the mixing of ultraviolet (UV) and infrared (IR) divergences reminiscent of the UV/IR connection of the string the-

ory. For the $\phi^{4}$ theory, it has been proved to remain ultraviolet renormalizable up to two loops [14], although this does not seem to hold to all order [12].

In this paper we will investigate the effective potential for the scalar field theory in the noncommutative spacetime. Our interesting is to see how the noncommutativity of the spacetime will affect the Coleman-Weinberg mechanism [17]. We will use the path-integration formulation [18] to evaluate the effective potential to the two-loop approximation. In this formulation it is easy to see that there is no nonplanar diagram in the one-loop potential. Thus the spontaneous symmetry breaking is blind to the noncommutativity at this level. However, we find that the nonplanar diagram can become dominant in the two-loop potential, if the noncommutativity of geometry is sufficiently small. We also find that the nonplanar diagram has an inclination to induce the spontaneously symmetry breaking if it is not broken in the tree level. It also has an inclination to restore the symmetry breaking if it has been broken in the tree level. 


\section{Formulation and one-loop diagram}

The noncommutative geometry we considered is defined by the coordinates $x_{\mu}$ which satisfy the relation

$$
\left[x_{\mu}, x_{\nu}\right]=i \theta_{\mu \nu}
$$

in which $\theta_{\mu \nu}$ is a real, antisymmetric matrix. The function defined over the noncommutative spacetime can be expressed as

$$
f(x)=\frac{1}{(2 \pi)^{4}} \int d^{4} k e^{i k_{\mu} x^{\mu}} \tilde{f}(k) .
$$

The Moyal product $(*)$ of two functions is then defined by [11-16]

$$
\begin{aligned}
f(x) * g(x) & =\frac{1}{(2 \pi)^{8}} \int d^{4} k d^{4} p e^{i k_{\mu} x^{\mu}} e^{i p_{\nu} x^{\nu}} \tilde{f}(k) \tilde{g}(p) \\
& =\frac{1}{(2 \pi)^{8}} \int d^{4} k d^{4} p e^{i\left(k_{\mu}+p_{\mu}\right) x^{\mu}-\frac{i}{2} k_{\mu} \theta^{\mu \nu} p_{\nu}} \tilde{f}(k) \tilde{g}(p) \\
& =\left.e^{+\frac{i}{2} \theta^{\mu \nu} \frac{\partial}{\partial y^{\mu}} \frac{\partial}{\partial z^{\nu}}} f(y) g(z)\right|_{y, z \rightarrow x} .
\end{aligned}
$$

We consider the $\phi^{4}$ theory with the action

$$
S[\phi]=\int d^{4} x \mathcal{L}(\phi)=\int d^{4} x\left[\frac{1}{2} \partial_{\mu} \phi * \partial^{\mu} \phi+\frac{1}{2} m^{2} \phi * \phi-\frac{\lambda}{4 !} \phi * \phi * \phi * \phi\right] .
$$

After expanding the Lagrangian around a constant field $\phi_{0}$ the action can be expressed as

$$
S[\phi]=S\left[\phi_{0}\right]+\left.\frac{1}{2} \int d^{4} x d^{4} y \tilde{\phi}(x) * \tilde{\phi}(y) \frac{\delta^{2} S}{\delta \phi(x) \delta \phi(y)}\right|_{\phi_{0}}+\int d^{4} x \tilde{\mathcal{L}_{\mathcal{I}}}\left(\tilde{\phi}, \phi_{0}\right),
$$

in which $\tilde{\phi} \equiv \phi-\phi_{0}$ and $\tilde{\mathcal{L}_{\mathcal{I}}}\left(\tilde{\phi}, \phi_{0}\right)$ can be found from the Lagrangian Eq.(2.4). (Note that

as the constant field $\phi_{0}$ is a stationary point we have the relation $\left.\frac{\delta S}{\delta \phi}\right|_{\phi_{0}}=0$.) Then using the propagator defined by

$$
\begin{gathered}
i D^{-1}\left(\phi_{0} ; k\right)=\int d^{4} k e^{i k x} i D^{-1}\left(\phi_{0} ; x\right), \\
i D^{-1}\left(\phi_{0} ; x, y\right)=\left.\frac{\delta^{2} S}{\delta \phi(x) \delta \phi(y)}\right|_{\phi_{0}},
\end{gathered}
$$


the effective potential $V\left(\phi_{0}\right)$ is found to be [18]

$$
V\left(\phi_{0}\right)=V_{0}\left(\phi_{0}\right)-\frac{1}{2} i \hbar \int \frac{d^{4} k}{(2 \pi)^{4}} \ln \operatorname{det} i D^{-1}\left(\phi_{0} ; k\right)+i \hbar<\exp \left(\frac{i}{\hbar} \int d^{4} x \tilde{\mathcal{L}_{\mathcal{I}}}\left(\tilde{\phi}, \phi_{0}\right)\right)>
$$

The first term in Eq.(2.7) is the classical potential. The second term is the one-loop contribution from the second term in Eq.(2.5). It is known that, as $\theta_{\mu \nu}$ is an antisymmetric matrix the quadratic part of the field $\phi(x)$ in the noncommutative spacetime will be identical with that in the commutative spacetime, after the spacetime integral [11-16]. Therefore, this elementary property implies that the Moyal product in the second term of Eq.(2.5) can be dropped. Thus we see an interesting property that the noncommutativity of spacetime dose not affect the potential in one-loop level. This property had been found by Campbell and Kaminsky [15] after investigating the tadpole diagram in the linear sigma model.

The third term in Eq.(2.7) is the higher-loop contribution of the effective potential. To obtain it we shall evaluate the expection value of the third term in Eq.(2.5) by the conventional Feynmam rule, with $D\left(\phi_{0} ; k\right)$ as the propagator and keep only the connected single-particle irreducible graphs [18]. In the next section we will analyze the two-loop diagram and find that the nonplanar diagram can become dominant if the noncommutativity of geometry is sufficiently small.

\section{Two-loop diagram}

In the two-loop level there are two planar and nonplanar diagrams. Using the Feynman rule, which includes the propagator

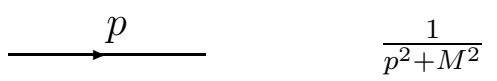

and vertices

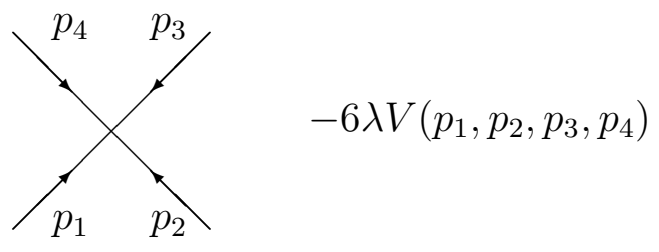




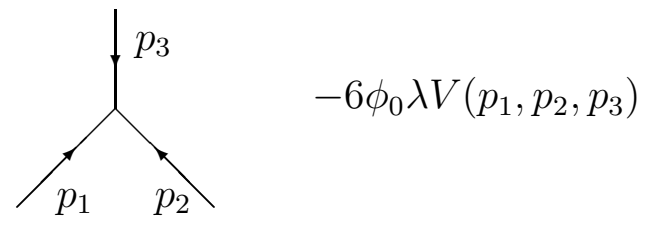

in which $M^{2} \equiv m^{2}+\frac{1}{2} \lambda \phi_{0}^{2}$ and $V\left(p_{i}\right)=e^{\frac{-i}{2} \sum_{i<j} p_{i \mu} \theta^{\mu \nu} p_{j \nu}}$, the contributions of the effective potential from the planar diagram are [18]

$$
\begin{aligned}
& =I_{1}^{P} \\
& =\frac{2}{3} \frac{\hbar^{2}}{24} \lambda \int \frac{d^{4} k}{(2 \pi)^{4}} \frac{1}{k^{2}+M^{2}} \int \frac{d^{4} p}{(2 \pi)^{4}} \frac{1}{p^{2}+M^{2}}, \\
& =I_{2}^{P} \\
& =-\frac{1}{2} \frac{\hbar^{2}}{36} \lambda^{2} \phi_{0}^{2} \int \frac{d^{4} k d^{4} p}{(2 \pi)^{8}} \frac{1}{\left(k^{2}+M^{2}\right)\left(p^{2}+M^{2}\right)\left((k+p)^{2}+M^{2}\right)} .
\end{aligned}
$$

The contributions of th effective potential from the nonplanar diagram are like those in the planar diagram, while with an extra factor $e^{i k_{\mu} \theta^{\mu \nu} p_{\nu}}$. They are

$$
\begin{gathered}
I_{1}^{N}=\frac{1}{3} \frac{\hbar^{2}}{24} \lambda \int \frac{d^{4} k d^{4} p}{(2 \pi)^{8}} \frac{e^{i k_{\mu} \theta^{\mu \nu}} p_{\nu}}{\left(k^{2}+M^{2}\right)\left(p^{2}+M^{2}\right)}, \\
I_{2}^{N}=-\frac{1}{2} \frac{\hbar^{2}}{36} \lambda^{2} \phi_{0}^{2} \int \frac{d^{4} k d^{4} p}{(2 \pi)^{8}} \frac{e^{i k_{\mu} \theta^{\mu \nu} p_{\nu}}}{\left(k^{2}+M^{2}\right)\left(p^{2}+M^{2}\right)\left((k+p)^{2}+M^{2}\right)} .
\end{gathered}
$$

Note that the factors $\frac{2}{3}\left(\frac{1}{3}\right)$ appearing in Eqs. (3.1) ((3.3)) means that the associated planar (nonplanar) diagram will be with $2 / 3(1 / 3)$ weight of the commutative graph. And factors $\frac{1}{2}$ appearing in Eqs. (3.2) and (3.4) means that the associated planar and nonplanar diagram will be both with $1 / 2$ weight of the commutative graph. The counting has been detailed by Campbell and Kaminsky [15] in investigating the linear sigma model. Let us describe it again for the completeness.

The diagram (3.1) has a single vertex, and so a phase factor $V(p, k,-k,-p)$. Of the six possible orderings (modulo cyclic permutation) of the set $\{p, k,-k,-p\}$, four lead to 
a trival phase factor, and two lead to a phase of either $e^{i k_{\mu} \theta^{\mu \nu} p_{\nu}}$ or $e^{-i k_{\mu} \theta^{\mu \nu} p_{\nu}}$ (which are the same under the integral over the loop momenta $k$ ). Thus the planar diagram will with $4 / 6=2 / 3$ weigth and the nonplanar diagram will with $2 / 6=1 / 3$ weigth with respect to the commutative graph. .

The diagram (3.2) has two vertices, and we pick up the phase factor $V(p, k,-p-$ $k) V(-k,-p, p+k)$. Each vertex has two orderings (modulo cyclic permutation), for four combinations total. Explicity evaluation will find that two lead to a trival phase factor, and two lead to a phase $e^{i k_{\mu} \theta^{\mu \nu} p_{\nu}}$. Thus the planar diagram will with $2 / 4=1 / 2$ weigth and the nonplanar diagram will with $2 / 4=1 / 2$ weigth with respect to the commutative graph.

The planar-diagram contributions of Eqs.(3.1) and (3.2) have ultraviolet divergences and shall be renormalized. This can be analyzed following that in [18]. However, due to the phase factor in Eqs.(3.3) and (3.4) the nonplanar-diagram contributions may be finite. We will analyze the nonplanar-diagram contributions in below.

Let us first analyze Eq.(3.3). Using the Schwinger parameters, $\alpha_{1}$ and $\alpha_{2}$, we see that

$$
\begin{gathered}
\int d^{4} k d^{4} p \frac{e^{i k_{\mu} \theta^{\mu \nu} p_{\nu}}}{\left(k^{2}+M^{2}\right)\left(p^{2}+M^{2}\right)}=\int_{0}^{\infty} d \alpha_{1} \int_{0}^{\infty} d \alpha_{2} \int d^{4} k d^{4} p e^{i k_{\mu} \theta^{\mu \nu} p_{\nu}} e^{-\alpha_{1}\left(k^{2}+M^{2}\right)} e^{-\alpha_{2}\left(p^{2}+M^{2}\right)} \\
=\int_{0}^{\infty} d \alpha_{1} \int_{0}^{\infty} d \alpha_{2} \int d^{4} p e^{-\frac{1}{4 \alpha_{1}} \tilde{p}_{\mu} \tilde{p}^{\mu}} e^{-\alpha_{2}\left(p^{2}+M^{2}\right)} \int d^{4} l e^{-\alpha_{1}\left(l^{2}+M^{2}\right)} \\
=\int_{0}^{\infty} d \alpha_{1} \int_{0}^{\infty} d \alpha_{2}\left(\frac{\pi}{\alpha_{1}}\right)^{2} e^{-\left(\alpha_{1}+\alpha_{2}\right) M^{2}} \int d^{4} p e^{-\frac{1}{4 \alpha_{1}} \tilde{p}_{\mu} \tilde{p}^{\mu}} e^{-\alpha_{2} p^{2}}
\end{gathered}
$$

in which $\tilde{p}^{\mu}=\theta^{\mu \nu} p_{\nu}$ and $l_{\mu}=k_{\mu}-\frac{i}{2 \alpha_{1}} \tilde{p}_{\mu}$. Next, we analyze Eq.(3.4). Using Feynman parameter $w$ and then the Schwinger parameters, $\alpha_{1}$ and $\alpha_{2}$, we see that

$$
\begin{aligned}
& \int d^{4} k d^{4} p \frac{e^{i k_{\mu} \theta^{\mu \nu} p_{\nu}}}{\left(k^{2}+M^{2}\right)\left(p^{2}+M^{2}\right)\left((k+p)^{2}+M^{2}\right)} \\
= & \int_{0}^{1} d w \int d^{4} k d^{4} p \frac{1}{\left[w\left(k^{2}+M^{2}\right)+(1-w)\left((k+p)^{2} M^{2}\right)\right]^{2}} \frac{e^{i k_{\mu} \theta^{\mu \nu} p_{\nu}}}{p^{2}+M^{2}} \\
= & \int_{0}^{1} d w \int d^{4} q d^{4} p \frac{1}{\left.\left[q^{2}+\left(w-w^{2}\right) p^{2}+M^{2}\right)\right]^{2}} \frac{e^{i q_{\mu} \theta^{\mu \nu} p_{\nu}}}{p^{2}+M^{2}} .
\end{aligned}
$$




$$
\begin{aligned}
& =\int_{0}^{1} d w \int_{0}^{\infty} d \alpha_{1} \int_{0}^{\infty} d \alpha_{2} \int d^{4} q d^{4} p \alpha_{1} e^{\left.-\alpha_{1}\left[q^{2}+\left(w-w^{2}\right) p^{2}+M^{2}\right)\right]} e^{i q_{\mu} \theta^{\mu \nu} p_{\nu}} e^{-\alpha_{2}\left(p^{2}+M^{2}\right)} \\
& =\int_{0}^{1} d w \int_{0}^{\infty} d \alpha_{1} \int_{0}^{\infty} d \alpha_{2} \int d^{4} p \alpha_{1} e^{-\frac{1}{4 \alpha_{1}} \tilde{p}_{\mu} \tilde{p}^{\mu}} e^{-\left(\alpha_{2}+\alpha_{1}\left(w-w^{2}\right)\right) p^{2}-\alpha_{2} M^{2}} \int d^{4} l e^{-\alpha_{1}\left(l^{2}+M^{2}\right)} \\
& =\int_{0}^{1} d w \int_{0}^{\infty} d \alpha_{1} \int_{0}^{\infty} d \alpha_{2} \frac{\pi^{2}}{\alpha_{1}} e^{-\left(\alpha_{1}+\alpha_{2}\right) M^{2}} \int d^{4} p e^{-\frac{1}{4 \alpha_{1}} \tilde{p}_{\mu} \tilde{p}^{\mu}} e^{-\left(\alpha_{2}+\alpha_{1}\left(w-w^{2}\right)\right) p^{2}},
\end{aligned}
$$

in which $q_{\mu}=k_{\mu}-(1-w) p_{\mu}, \tilde{p}^{\mu}=\theta^{\mu \nu} p_{\nu}$ and $l_{\mu}=q_{\mu}-\frac{i}{2 \alpha_{1}} \tilde{p}_{\mu}$. To evaluate Eqs.(3.5) and (3.6) furthermore we shall know the exact form of $\tilde{p}_{\mu}$ which depends on the noncommutativity parameters $\theta_{\mu \nu}$.

Denoting the noncommutativity parameters as

$$
\theta_{\mu \nu}=\left[\begin{array}{cccc}
0 & a & b & c \\
-a & 0 & d & e \\
-b & -d & 0 & f \\
-c & -e & -f & 0
\end{array}\right]
$$

then $\tilde{p}_{\mu} \tilde{p}^{\mu} \equiv p^{\nu} \theta_{\mu \nu} \theta^{\mu \lambda} p_{\lambda} \equiv p^{\nu} U_{\nu}^{\lambda} p_{\lambda}$ where $U_{\nu}^{\lambda}$ is a symmetric matrix. Because that any real, symmetric matrix can be diagonalized by an orthogonal matrix we can thus change the orthonormal variables $p_{\mu}$ to another orthonormal variables $h_{\mu}$ such that $\frac{1}{4 \alpha_{1}} \tilde{p}_{\mu} \tilde{p}^{\mu}+\alpha_{2} p_{\mu} p^{\mu}=$ $\lambda_{1} h_{1}^{2}+\lambda_{2} h_{2}^{2}+\lambda_{3} h_{3}^{2}+\lambda_{4} h_{4}^{2}$, where $\lambda_{i}$ been the eigenvalues of the associated matrix. The eigenvalues are found to be

$$
\lambda_{1}=\lambda_{2}=\alpha_{2}+\frac{1}{8 \alpha_{1}}\left(S+\sqrt{S^{2}-4 D^{2}}\right), \quad \lambda_{3}=\lambda_{4}=\alpha_{2}+\frac{1}{8 \alpha_{1}}\left(S-\sqrt{S^{2}-4 D^{2}}\right)
$$

where

$$
S \equiv a^{2}+b^{2}+c^{2}+d^{2}+e^{2}+f^{2}, \quad D \equiv c d-b e+a f .
$$

Thus, after the integration of $h_{\mu}$ Eqs. (3.5) becomes

$$
\int_{0}^{\infty} d \alpha_{1} \int_{0}^{\infty} d \alpha_{2} \frac{8 \pi^{2}}{S+\sqrt{S^{2}-4 D^{2}}+8 \alpha_{1} \alpha_{2}} \frac{8 \pi^{2}}{S-\sqrt{S^{2}-4 D^{2}}+8 \alpha_{1} \alpha_{2}} e^{-\left(\alpha_{1}+\alpha_{2}\right) M^{2}} .
$$


In a similar way, Eqs. (3.6) becomes

$$
\begin{array}{r}
\int_{0}^{1} d w \int_{0}^{\infty} d \alpha_{1} \alpha_{1} \int_{0}^{\infty} d \alpha_{2} \frac{8 \pi^{2}}{S+\sqrt{S^{2}-4 D^{2}}+8 \alpha_{1}\left(\alpha_{2}+\left(w-w^{2}\right) \alpha_{1}\right)} \\
\times \frac{8 \pi^{2}}{S-\sqrt{S^{2}-4 D^{2}}+8 \alpha_{1}\left(\alpha_{2}+\left(w-w^{2}\right) \alpha_{1}\right)} e^{-\left(\alpha_{1}+\alpha_{2}\right) M^{2}}
\end{array}
$$

It is easy to see that the above two relations will become divergent if $S=D=0$. This is because that the factors $\frac{1}{8 \alpha_{1} \alpha_{2}}$ and $\frac{1}{8 \alpha_{1}\left(\alpha_{2}+\left(w-w^{2}\right) \alpha_{1}\right)}$ is divergent at $\alpha_{1}=0$ and (or) $\alpha_{2}=0$. Therefore when the noncommutativity parameter $\theta$ is very small we can approximate the above relations by

$$
\begin{aligned}
& (3.10 a) \approx \int_{0}^{\infty} d \alpha_{1} \int_{0}^{\infty} d \alpha_{2} \frac{16 \pi^{4}}{D^{2}} e^{-\left(\alpha_{1}+\alpha_{2}\right) M^{2}}=\frac{16 \pi^{4}}{D^{2} M^{4}} \\
& (3.11 a) \approx \int_{0}^{\infty} d \alpha_{1} \int_{0}^{\infty} d \alpha_{2} \alpha_{1} \frac{16 \pi^{4}}{D^{2}} e^{-\left(\alpha_{1}+\alpha_{2}\right) M^{2}}=\frac{16 \pi^{4}}{D^{2} M^{6}}
\end{aligned}
$$

Substituting the above results into Eqs.(3.3) and (3.4) we finally find the nonplanardiagram contribution of the effective potential

$$
V\left(\phi_{0}\right)^{\text {nonplanar }}=\lambda \frac{\hbar^{2}}{72} \frac{16 \pi^{4}}{D^{2} M^{4}}\left(1-\frac{\lambda \phi_{0}^{2}}{M^{2}}\right)
$$

Thus we see that the nonplanar diagram can become dominant in the two-loop potential, if the noncommutativity of geometry is sufficiently small.

Note that the inverse power of $D^{2}$ in the Eq.(3.12) is a consequence of dimensional analysis, coupled with the fact that the diagrams of Eqs.(3.1) and (3.2) have degree of 
divergence 4 . This could guarantee that the higher loop graphs are not more singular than $\frac{1}{D^{2}}$ (up to logarithmic corrections), whereas they will appear with higher powers of $\lambda$. Thus, if $D$ is chosen to be of order $\lambda$, the two loop diagram will dominate over higher loop diagram (and lower loop diagrms) and the calculations of this paper become useful in determining the effects of quantum correction on the symmetry property. The details of a very similar argument have presented in reference 13.

From Eq.(3.13) we have the relation

$$
\frac{\partial V\left(\phi_{0}\right)^{\text {nonplanar }}}{\partial\left(\phi_{0}^{2}\right)}=\lambda^{2} \frac{\hbar^{2}}{9} \frac{\pi^{4}}{D^{2} M^{8}}\left(\lambda \phi_{0}^{2}-4 m^{2}\right) .
$$

Therefore, if $m^{2}>0$, i.e., the symmetry is not broken in the tree level, then from Eq.(3.13) we see that the value $\partial V\left(\phi_{0}\right)^{\text {nonplanar }} / \partial\left(\phi_{0}^{2}\right)$ becomes negative if $\phi_{0}^{2}$ is small. This means that the nonplanar diagram has an inclination to induce spontaneously symmetry breaking if it is not broken in the tree level. On the othe hand, if $m^{2}<0$, i.e., the symmetry has been broken spontaneously broken in the tree level, the value $\partial V\left(\phi_{0}\right)^{\text {nonplanar }} / \partial\left(\phi_{0}^{2}\right)$ in Eq.(3.13) is positive definitively. This means the nonplanar diagram has an inclination to restore the symmetry breaking if it has been broken in the tree level.

It is difficult to analyze the system with $D=0$ (note that $S$ defined in Eq.(3.9) is positive), but we belive that the above property will not be changed. The problem is remained in futher investigation.

\section{Conclusion}

In this paper we have evaluated the renormalized effective potential for the scalar field theory in the noncommutative spacetime to the two-loop approximation. In the path-integration formulation we see that there is no nonplanar diagram in the one-loop potential. Thus the spontaneous symmetry breaking is blind to the noncommutativity at this level. However, we find that the nonplanar diagram can become dominant in the two-loop potential, if the noncommutativity of geometry is sufficiently small. We also find that the nonplanar diagram 
has an inclination to induce spontaneously symmetry breaking if it is not broken in the tree level. It also has an inclination to restore the symmetry breaking if it has been broken in the tree level.

Finally, as the U(1) Yang-Mills field will couple to itself in the noncommutative spacetime $[11,16]$ the new Feynmam diagram shall be involved in evaluating the effective potential. It is interesting to see how the new diagram and the noncommutativity of the spacetime will affect the Coleman-Weinberg mechanism in the theory including the Yang-Mills field. Work on the problem is in progress. 
1. H. S. Snyder, Phys. Rev. 71 (1947) 38; 72 (1947) 68.

2. Connes, Noncommutative Geometry Academic. Press, New York, 1994); Connes A.Gravity coupled with matter and the foundation of non commutative geometry, Comm. in Math. Phys. 182 155-177 (1996), hep-th/9603053.

3. Landi G. An introduction to noncommutative spaces and their geometries, Lecture Notes in Physics, Springer-Verlag, hep-th/97801078; Várilly J. An introduction to noncommutative geometry, Summer School "Noncommutative geometry and applications", Lisbon, September 1997 , physics/97090045

4. Schücker T. Geometries and forces, Summer School "Noncommutative geometry and applications", Lisbon September 1997 , hep-th/9712095.

5. T. Filk, Phys. Lett. B 376 (1996) 53; see also Chris P. Korthals-Altes and A. GonzalezArroyo, Phys. Lett. 131B (1983) 396.

6. A. Connes, M. R. Douglas and A. Schwarz, "Noncommutative Geometry and Matrix Theory: Compactification on Tori", hep-th/9711162, JHEP 9802:003 (1998).

7. B. Morariu and B. Zumino, "Super Yang-Mills on the Noncommutative Torus," hepth/9807198; C. Hofman and E. Verlinde, "U-duality of Born-Infeld on the Noncommutative Two-Torus," hep-th/9810116, JHEP 9812, 010 (1998)

8. T. Krajewski and R. Wulkenhaar, "Perturbative Quantum Gauge Fields on the Noncommutative Torus," hep-th/9903187; M. M. Sheikh-Jabbari, "Renormalizability of the Supersymmetric Yang-Mills Theories on the Noncommutative Torus", hep-th/9903107, JHEP 9906, 015 (1999).

9. N. Seiberg and E. Witten, "String Theory and Noncommutative Geometry," hepth/9908142, JHEP 9909, 032 (1999).

10. N. A. Obers and B. Pioline, Phys. Rep. 318 (1999) 113. 
11. C. P. Martin and D. Sanchez-Ruiz, "The One-loop UV Divergent Structure of U(1) Yang-Mills Theory on Noncommutative $R^{4}$," hep-th/9903077, Phys. Rev. Lett. 83, 476 (1999)

12. I. Chepelev and R. Roiban, "Renormalization of Quantum field Theories on Noncommutative $R^{d}$. I: Scalars," hep-th/9911098.

13. S. Minwalla, M. Van Raamsdonk and N. Seiberg, "Noncommutative Perturbative Dynamics," hep-th/9912072, JHEP 0002, 020 (2000).

14. I. Y. Aref'eva, D. M. Belov and A. S. Koshelev, "Two-loop Diagrams in Noncommutative $\phi_{4}^{4}$ Theory", hep-th/9912075; A. Micu, "Noncommutative $\phi^{4}$ Theory at Two loop", hep-th/0008057.

15. B. A. Campbell and K. Kaminsky, Nucl. Phys. B 581 (2000) 240, hep-th/0003137.

16. A. Matusis, L Ausskind and N. Toumbas, "The IR/UV Connection in Noncommutative Gauge Theories,"hep-th/0002075; M. Hayakawa, "Perturbative Analysis on Infrared and Ultraviolet Aspects of Noncommutative QED on $R^{4}$," hep-th/9912167; "Perturbative Analysis on Infrared Aspects of Noncommutative QED on $R^{4}$," hep-th/9912094.

17. S. Coleman and E. Weinberg, Phys. Rev. D 7 (1973) 1888.

18. R. Jackiw, Phys. Rev. D 9 (1974) 1686. 